\title{
La recuperación de información automatizada: sus reorientar posibilidades para algunas funciones bibliotecarias
}

\author{
Fernando Edmundo González*
}

\section{RESUMEN}

Se presen tan al gu nas con si de ra cio nes que giran en tor no ala confec ción de ca tálogos elec tró ni cos. Asi mis mo, se exa mi na de qué forma el em pleo de una com pu ta do ra al te ra o mo di fi ca la crea ción de un ca tá lo go y de cómo esto tiene re percu sio nes en el ni vel de servicios que ofrece la biblioteca.

\begin{abstract}
Certain con si de ra tions are pre sen ted that re vol ve around the creation of elec tro nic catalogues. Li kewi se, an exa mi nation is made of how the em plo yment of a com pu ter al ters or mo di fies the crea tion of a ca ta lo gue and h ow this has re per cus sions upon the le vel of services that the library offers.
\end{abstract}

\section{INTRODUCCION}

A par tir de la dé ca da de los ochen ta el uso de las mi cro com putadoras se generalizó en la sociedad mexicana, gracias primor dial men te a su bajo cos to y a la dis po ni bi li dad de pro gramas tales como procesadores de texto, hojas electrónicas, manejadores de bases de datos, etc.

En los primeros años de la introducción de las computadoras per so na les la ma yo ría de las bi blio te cas se man tu vie ron al margen ya que sólo existía software de aplicación general; pero con el paso del tiempofueron desarrolladasy/oin tro ducidasal país aplicaciones modulares e integrales para bibliotecas, lo que pro vo cóel in te rés de los bi bliote ca rios por au to ma ti zar sus procesos. En trelos primeros sistemas nacionalesdesarrollados para bibliotecas tenemos a LogiCat, SIABUC y BIBLOS.

En la actualidad se ha vuelto común enterarse del inicio de proyectos de automatización de bibliotecas: el uso de CD-ROM con acervos bibliográficos, el desarrollo de software de ges tión bi blio te ca ria, la crea ción de ba ses de da tos bibliográficas, etc. En el medio bibliotecario se respira y vive un am bien te en don de el de sa rro llo de ca tálo gos elec tró ni cos es la mira hacia la cual tienden muchas bibliotecas.

Lo anterior se debe en gran medida a que existe la opinión, muy extendida, de que crear un catálogo en línea, en sí mismo, so lu cio na to dos o casi to dos los pro ble mas de una bi bliote ca, v. gr., re za go de ma te rial por pro ce sar, agi li za ción de la recuperación de información, etc.

Convendría tener en cuenta la advertencia que nos hace David Kelley: "si bien las mi cro com pu ta do ras cier ta men te pueden revolucionar los servicios bibliotecarios, no constituyen en sí una pa na cea." ${ }^{(1)}$ En el mis mo te nor Tom Nor ton nos aler- ta so bre el he cho de mi ni mi zar las des ven tajas de la au to ma tiza ción de los pro ce sos bi blio te ca rios: "hoy en día los sis te mas computarizados presentan excitantes posibilidades en el trabajobibliotecario pero es importante no menospreciar sus limitaciones..."(2)

En contraparte a lo anterior, algunos bibliotecarios han cuestionado que se utilicen las microcomputadoras para realizar los procesos automatizados de la misma forma como se llevan a cabolos proce sos manuales, de sa provechan do o subutilizando los recursos que pone a nuestro alcance el procesamiento electrónico de información..$^{(3)}$ Al respecto Wilson Sthal se ña la que "la opor tu ni dad que los sis te mas au to ma ti zados ofre cen a las bi blio te cas para me jo rar y ex pan dir sus servicios es inmejorable, pero para poder aprovechar esta oportunidad es necesario reorientar algunas actividades bibliotecarias para que permitan ofrecer nuevos niveles de servicios". (4)

Ante la polémica anterior es conveniente partir del hecho de que si bien el ca tá lo go es una he rra mien ta bá si ca del bi blio tecario, ya que permite la identificación completa de un documento diferenciándolo de los demás documentos que integran el acervo, su uso necesariamente varía si se trata de un catálogo convencional o de uno en línea.

Mientras que en un catálogo tradicional la búsqueda exige todo del sujeto, en un catálogo electrónico la búsqueda es de tipo interactivo, en donde se requiere que el interrogador (usuario) preconfigure mentalmente una pregunta (simple o com pues ta) y pos te rior men te la so me ta al sis te ma; con la respues ta del sis te ma se ini cia rá un pro ce so que con clui rá al obte ner el usua rio las re fe ren cias de los do cu men tos que le sean de uti li dad, ade más el ca tálo go elec trónicocuen ta con ca pacidades dein tercalación, combinación, comparación y or dena-

* Investigador del Centro Universitario de Investigaciones Bibliotecológicas 
ción, que permiten darle variados tratamientos a la información recuperada.

Lo importante de la controversia en tor no al de sa rro llo de catálogos electrónicos es el definir de qué forma el empleo de unamicrocom putadoraal tera omo dificalacreación y utilización de un catálogo.

\section{GENERACION DE ESTRUCTURAS DE DATOS}

$\mathrm{Al}$ abrir las bi blio te cas sus puer tas a las com pu ta do ras die ron paso a una nueva tecnología para el tratamiento documental de los acervos basada en la generación de estructuras de datos.

Un dato es un atributo de al gún ob je to o en ti dad.En re lación con un li bro los da tos se rían el au tor, tí tu lo, edi ción, pie de imprenta y los temas. A su vez, una estructuraes ladescripción de los elementos de un sistema y el tipo de conexiones entre ellos.

Las es truc tu ras de da tos res pon den ala des crip ción ge ne ral de loselementos-da to que in te granunre gistro. Es tas estructuras presentan una organización lógica de los datos, sus vinculaciones internas y las formas como se relacionan cada uno de los elementos del registro.

La estructura de datos se constituye por un identificador del dato (etiqueta) y la representación documental que a su vez representa el dato. Este es un esquema normalizado para el manejo de bases de da tos bi blio gráficas del cual se ha par ti do para el de sa rrollo de los for ma tos para la lec tu ra por com pu tadora de información catalográfica.

En el formato MARC (Machine Readable Cataloging) cada uno de los elementos de la ficha catalográfica tiene asignado un identificador numérico de 3 cifras, v. gr.:

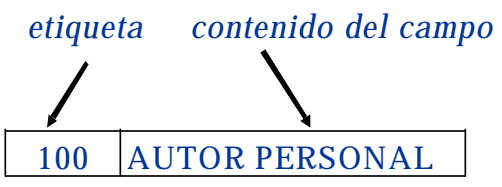

\begin{tabular}{|l|l|}
\hline 240 & TITULO \\
\hline
\end{tabular}

\begin{tabular}{|c|c|c|c|}
\hline 250 & \multicolumn{3}{|c|}{ EDICION } \\
\hline & 260 & \multicolumn{2}{|c|}{ PIE DE IMPRENTA } \\
\hline & & 600 & ENCAB. DE \\
\hline
\end{tabular}

De forma similar, en el formato CCF (Commom Communication Format) a cada elemento catalográficole corresponde un identificador numérico, v. gr.:

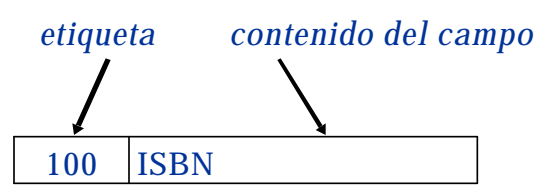

\begin{tabular}{|l|l|}
\hline 200 & TITULO \\
\hline
\end{tabular}

\begin{tabular}{|l|l|}
\hline 260 & EDICION \\
\hline
\end{tabular}

\begin{tabular}{|c|c|c|}
\hline 300 & \multicolumn{2}{|c|}{ AUTOR } \\
\hline & 400 & LUG., PUB. Y EDIT \\
\hline
\end{tabular}

Dentro de ambos formatos cada campo cuenta con indicadores que especifican el contenido del campo, códigos de subcam po queiden tifican un ele men to particular del cam poy delimitadores que señalan la localización del campo dentro del registro.

Algunos de los sistemas de gestión bibliotecaria para microcomputadorasutilizanlaestructuraanterior(etiqueta-campo) para crear los archivos que integrarán las bases de datos. Otros sistemas han creado diversas estructuras.

Des de un prin ci pio, los for ma tos de au to ma ti za ción de in formación catalográfica se ajustaron a la descripción catalográfica establecida en las normas Anglo-American Cataloguing Rules (AACR, 1967), ${ }^{(5)}$ las cuales en su segunda edición (1978) "ratifican su compatibilidad conel for mato MARC".

La descripción catalográfica se realiza con base en determina das re glas de ca ta lo ga ción, sien do las AACR 2 ed. el có digo más extendido e influyente.

Las re glas AACR2 se di vi den en: 1) des crip ción de los do cumen tos y,2) elec ción de los pun tos de ac ce so (en ca be za mientos de materia) y las entradas de las fichas secundarias. Las mismas reglas de descripción documental se siguen en los procesos de catalogación automatizada.

Dado que las re glas parahacerca tálogos si guen vi gen testanto para la catalogación manual como para la automatizada, y que los formatos más utilizados para la representación automatizadadere gis troscatalográficos res pondenalas AACR2, podemos afirmar que el empleo de la computadora en tareas deorganización de material documental no implica la alteración de los mé to dos de descrip ción catalo gráfica y organización de los catálogos, pero sí altera el procesamiento de los da tos en la me di da en que nos ofre ce po si bi li da des de cóm puto (comparación, intercalación, combinaciones y ordenaciones de los datos).

\section{RECUPERACION DE INFORMACION}

Las fun cio nes bási cas que de sa rro llan los sis te mas ca ta lo gráficos automatizados son el almacenamiento, actualización y 
recuperación de información. La tercera función está determinada por las dos primeras.

La recuperación de información es un proceso que realiza el usuario con el sistema, el cual está orientado a identificar aquellos re gis tros (docu men tos) que para el usua rio sean re levantes.

En un catálogo convencional los datos susceptibles de recuperarse son autor, título y los encabezamientos de materia. Este es que ma de re cu pe ración tra di cional ha sido trans por tado a los sistemas automatizados de gestión bibliotecaria.

Ha rold Borko se ña la esta si tua ción di cien do que "se ha transfor ma do el ca tálogo de tar je tas en unabase de da tos sin al te rar sucontenido".(7) Es de cir, el vie jo ca tálo go ha sido re em plazado por el catálogo electrónico pero sin modificar los esquemas de recuperación.

Se le ha dado una nue va pre sen ta ción al ca tá lo go, se han ob teni do nue vas ven ta jas ta les como la pro duc ción au to mática de reportes, tarjetas, etiquetas y la actualización inmediata de la base de da tos (pres ta cio nes con las que al gu nos biblio te ca rios se dan por satisfechos), pero la recuperación de información se ha visto favorecida mínimamente.

En un sis te ma ma nual la ma yor par te de las bús que das se reali zan so bre al gún tema que el usua rio co no ce o de sea co nocer, lo que implica determinarprimero la ma te ria o asun to de que se trata. Al respecto Robert Scarpit señala que "(el usuario) sólo en cuen tra lo que bus ca en el ca tá lo go cuan do lo haiden tificado previamente y esto es lo más difícil”."(8)

Esta si tuación se re pi te en los ca tálogos au to ma ti za dos o en lí nea en donde se tiene que realizar un proceso de aproximación de ensayo-error hasta recuperar aquellos documentos que sean relevantes para el usuario.

Lo anterior es debido a que los principales puntos de acceso para llegar al contenido de un libro son extremadamente inflexibles. Los encabezamientos de materia son la forma más ele men tal dere pre sentarel con te ni do de un docu men to, basados en criterios de organización de palabras según una sintaxispreestablecida; es de cir, que me dian te su em pleo se es peci fíca el len gua je de in te rro ga ción. Si no se si gue o co no ce la ló gica de este es que ma de sig nos pre co or dina dos la recuperación de información se verá limitada.

Con el uso de las microcomputadoras se ha reconsiderado el empleodesistemas postcoordinados ${ }^{(9)}$ (in dización, key word) los cua les com bi nan los tér mi nos al mo men to de las bús quedas. El principal in con venien te de esta for ma de re cu pe ración es el fenómeno ruido- silencio que se produce por imprecisión en las búsquedas.
Para dis mi nuir este efec to se uti li zan ope ra do res ló gi cos y rela cio na les (los cua les de fi nen la ope ra ción que se va a rea li zar entre dos o más elementos), el truncamiento de tér mi nos y la búsqueda sobre determinados campos.

Resulta evidente que el empleo de técnicas de postcoordinación de términos supera los beneficios obtenidos con los esquemas tradicionales, lo cual no significa que aquí se agoten todas las posibilidades.

El análisisdocumental o de contenido produce con el tiempo un vo ca bu la rio que se cons ti tui rá so bre la base de un len guaje documental.

Poder disponer de un lenguaje documental permite atenuar los efec tos de la he te ro ge nei dad al in di zar y re cu pe rar un documento. Como dice Antonio García Gutiérrez, "el empleo de códi gosidén ti cos por par te de los dis tin tos ele men tos compo nen tes del diálo go do cu men tal pro du ci rá una máxi ma pertinencia en la recuperación". ${ }^{(10)}$

Como se puede observar, la recuperación automática de infor mación con lle vaelem pleo de es que mas de re pre sen tación de con te ni do fle xi bles que per mi ten que el có di go asig na do a un do cu men to du ran te el análi sis coin ci da con el có di go utilizado en la bús que da, de tal for ma que la dis tor sión pro du ci da por el fenómeno ruido-silencio se reduce al mínimo.

Por eso es importante destacar el hecho de que en la confección de ca tálo gos au to ma tiza dos al gu nas téc nicas de des cripción documental han sido rebasadas por la tecnología disponible. No hay que olvidar que "la utilidad del catálogo debe ser evaluada en función de los requerimientos de los usuarios". (11)

\section{CONCLUSIONES}

La au to ma ti zación nos ofre ce la opor tu ni dad de re orien tar los objetivos de algunas funciones bibliotecarias.

Si bien la organización documental no se ve alterada por el em pleo de las com pu ta do ras, la co mu nicación de di cha in formación sí presenta restricciones debido al empleo de esquemas de recuperación limitados.

Un catálogo automatizado no se puede limitar a la información con te ni daen tar je tas de car tón, su de sa rro llo im plicanecesariamente un cambio de ideas y objetivos.

En este cam bio lo más di fícil no es ini ciar la tran si ción a nuevas for mas de re pre sen tar y re la cio nar el con te ni do de los documentos, sino el modificar estructuras mentales que se han consolidado a través de un quehacer caracterizado por el pragmatismo. 
NOTAS

(1) Kelley, David. "Software: what's available" en Microcomputers in libraries. Edited by Chingchih Chen and Stacey E. Bressler. New York: Neal-Schuman Publishers, 1983. p. 72.

(2) Nor ton, Tom. "Com puteri sation: a new di men sion for library and in for mation ser vices" en Procce dings on a Confe rence of the AS LIB Biolo gical and Agricul tu ralheld at the Science Group Commonwealth Institute London, June 9 1982. Editor H. L. Williams. London: ASLIB, 1983. p.13.

(3) Olson, Nancy B. "His tory of or ga nizing mi crocom puter soft ware" en Thelibrarymicrocomputers enviroment:management issues. Edi ted by Shei la S. Int ner and Jane Anne Han ni gan. New York: The ORIX Press, 1988. p. 23.

(4) Sthal, Wilson. "Riding the technology express: the causes and costs of system upgrades" en $A d$ vances in Automation and Net working. Edi tor Joe Hewitt. Lon don: Jai Press, 1988. vol. 2 p. 157.

(5) Morales Campos, Estela. El formato MARC en la automatización de información bibliográfica. México: UNAM. Dirección General de Bibliotecas, 1981. p. 89.

(6) Rowley, J. E. Computers for libraries. 2ed. London: Clive Bingley Ltd., 1985. p. 63

(7) Borko, Harold. "Information te chno logy: a look toward the fu tu re." en The library microcomputers enviroment: management issues. Edited by Sheila S. Intner and Jane Anne Hannigan. New York: The ORIX Press, 1988. p. 242.

(8) Escarpit, Robert. Teoría general de la información y de la comunicación. 2ed. Barcelona: ICARIA, 1981. p.

(9) El sistema UNITERM (1955) fue el primero de una generación de sistemas postcoordinados.

(10) García Gu tié rrez, An to nio. "Los len guajes do cu men ta les" en Fundamentos de información y documentación. José López Yepes comp. Madrid: EUDEMA, 1989. p. 324.

(11) Rowley Ibidem. p. 149. 